\title{
Functional assembly of two membrane-binding domains in listeriolysin 0 , the cytolysin of Listeria monocytogenes
}

\author{
Iharilalao Dubail, Nicolas Autret, Jean-Luc Beretti, Samer Kayal, \\ Patrick Berche and Alain Charbit
}

Laboratoire de

Microbiologie, INSERM

U-411, Faculté de Médecine Necker-Enfants Malades,

156 rue de Vaugirard, 75730 Paris Cedex 15, France
Author for correspondence: Alain Charbit. Tel: +331406153 76. Fax: +33140615592. e-mail: charbit@necker.fr

\begin{abstract}
Listeriolysin $\mathrm{O}$ (LLO) is a major virulence factor secreted by the pathogenic Listeria monocytogenes and acts as pore-forming cytolysin. Based on sequence similarities between LLO and perfringolysin (PFO), the cytolysin from Clostridium perfringens of known crystallographic structure, two truncated LLO proteins were produced: LLO-d123, comprising the first three predicted domains, and LLO-d4, the last C-terminal domain. The two proteins were efficiently secreted into the culture supernatant of $L$. monocytogenes and were able to bind to cell membranes. Strikingly, when expressed simultaneously, the two secreted domains LLO-d123 and LLO-d4 reassembled into a haemolytically active form. Two in-frame linker insertions were generated in the hinge region between the $\mathrm{d} 123$ and $\mathrm{d} 4$ domains. In both cases, the insertion created a major cleavage site for proteolytic degradation and abolished cytolytic activity, which might suggest that the region connecting d123 and d4 participates in the interaction between the two portions of the monomer.
\end{abstract}

Keywords: linker insertions, truncated proteins, domain interactions, LLO mutants

\section{INTRODUCTION}

Listeria monocytogenes is a Gram-positive bacterium that is widespread in nature and responsible for sporadic severe infections in humans and other animal species (Berche et al., 1987 and references therein). This pathogen is a facultative intracellular micro-organism, capable of invading most host cells, including epithelial cells (Gaillard et al., 1987), hepatocytes (Dramsi et al., 1995; Gaillard et al., 1996), fibroblasts (Kuhn \& Goebel, 1989), endothelial cells (Drevets et al., 1995) and even macrophages (Mackaness, 1962). Each step of the intracellular parasitism by L. monocytogenes is dependent upon the production of virulence factors (Sheehan et al., 1994). Among the virulence factors identified so far (Chakraborty et al., 1992; LeimeisterWachter et al., 1990; Renzoni et al., 1999), listeriolysin $\mathrm{O}$ (LLO; encoded by the hly gene) plays a crucial role in the escape of bacteria from the phagosomal compartment. Disruption of hly in wild-type L. monocytogenes

Abbreviations: HRBC, horse red blood cells; LLO, listeriolysin O; PFO, perfringolysin. leads to a loss of haemolytic activity and of virulence in the mouse model of infection (Gaillard et al., 1986; Kathariou et al., 1987; Portnoy et al., 1988).

LLO belongs to the family of thiol-activated cytolysins that are secreted by a large number of pathogenic Grampositive bacteria. These pore-forming toxins comprise more than 20 members to date (Bayley, 1997; Alouf, 2000), including the extensively studied perfringolysin (PFO) from Clostridium perfringens, streptolysin $\mathrm{O}$ from Streptococcus pyogenes and pneumolysin from Streptococcus pneumoniae. LLO is composed of 529 residues and possesses at its $\mathrm{N}$ terminus a 25 residue typical signal sequence (Mengaud et al., 1988). The protein is secreted into the culture supernatant as a monomer (Geoffroy et al., 1989). The three-dimensional structure of LLO is currently unknown but that of monomeric PFO has been determined at $2.7 \AA$ by X-ray crystallography (Rossjohn et al., 1997). The molecule, which comprises 500 residues, is elongated and is composed of four domains that are rich in $\beta$-sheet structures. Domain 2 is connected to domain 4 through a glycine linker (at residue 392). The autonomous domain 4 folds into a compact $\beta$ sandwich. Electron 
microscopy data showed that the PFO monomer is $\mathrm{L}$ shaped with four domains of equal size with one end, domain 4 (d4), flexibly linked to the three others.

A classical approach to understanding how the different regions of a polypeptide interact to stabilize the secondary, tertiary and quaternary structures of the native conformation consists of expressing these regions independently. Fragment complementation has been performed either with fragments produced by limited proteolysis or chemical cleavage, or with incomplete polypeptide chains expressed independently by genetic manipulations. The in vivo assembly of functional proteins from complementing fragments has been demonstrated for several proteins of Gram-negative bacteria, including integral membrane proteins (Bibi \& Kaback, 1990) and soluble cytoplasmic, periplasmic or secreted proteins (Betton \& Hofnung, 1994; Diep et al., 1998; Shiba \& Schimmel, 1992).

Taking advantage of the sequence similarities between LLO and PFO, we elaborated a theoretical 3D model of LLO folding, and engineered truncated and modified LLO proteins and expressed them in L. monocytogenes. We found that, when secreted simultaneously, two truncated proteins comprising the proximal (LLO-d123) and distal (LLO-d4) portions of LLO could reassemble to form an active molecule. In contrast, in-frame insertions in the region connecting these two domains drastically altered protein stability and abolished cytolytic activity.

\section{METHODS}

Bacterial strains and culture conditions. The wild-type virulent strain of $L$. monocytogenes EGD (denoted EGDwt) belongs to serovar $1 / 2 \mathrm{a}$ (15). EGD $\Delta$ hly is a derivative of EGDwt (serotype 1/2a) which contains an in-frame chromosomal deletion of $1080 \mathrm{bp}$ in the hly gene (Guzman et al., 1995). EGD $\Delta$ hly was transformed with the different recombinant plasmids by electroporation as previously described by Park \& Stewart (1990). Bacteria were grown in Brain Heart Infusion (BHI) broth (Difco Laboratories) at $37^{\circ} \mathrm{C}$ without antibiotics, except the pAT28-transformed strains which were grown on BHI broth containing $60 \mu \mathrm{g}$ spectinomycin $\mathrm{ml}^{-1}$.

Constructions. PCR-amplified fragments were first cloned into pCR (Invitrogen). All the recombinant genes were finally subcloned into the high-copy-number Gram-negative/Grampositive shuttle plasmid pAT28 (Trieu-Cuot et al., 1990). The wild-type hly gene preceded by its upstream promoter region, cloned into pAT28, has been described previously (Dubail et al., 2000).

LLO-d123. The truncated bly gene encoding the first 416 residues of LLO and its upstream promoter region were amplified by PCR using primers 1 (5'-CCGGATCCCTTAAAGTGACTTTTATGTTGAGGCA-3') and 2 (5'-CCCTGCAGTTAATCTGTATAAGCTTTTGAAGTTGTTTC-3'). The amplified product was first cloned into pTCV-lac and then subcloned into pAT28, yielding plasmid pAT28-LLOd123. The corresponding protein was named LLO-d123.

LLO-d4. To allow secretion of the predicted d4 domain of LLO into the culture medium, we fused residues $415-529$ to the proximal portion of the protein (residues 1-35) which contains the putative signal sequence. Two pairs of oligonucleotides were used for PCR amplification. The first pair of primers was 5'-GCTCTAGATCTCTTAAAGTGACTTTTATGTTGAGGCA-3' (primer 1) and 5'-GGGAATTCCATATGTGAATTTTCTTTATTGAATGCAGATGCATCCTTTGCTTCAGTTTG 3' (primer 2). An NdeI site (underlined) was created at the $3^{\prime}$ end directly after the triplet encoding residue 35. The second pair of primers was 5'-GGCCATATGACAGATGGAAAAATTAACATCGATCACTCT-3' (primer 1) and $5^{\prime}$-CCCTGCAGACAATTATTCGATTGGATTATCTAC-3' (primer 2). The two amplified fragments were cloned into pCR. An XbaI-KpnI fragment, comprising the proximal part of $h l y$, was first subcloned into the XbaI-KpnI sites of pAT28 (yielding pAT28-LLO:1-35). Then, an NdeI-KpnI fragment comprising the distal part of hly was inserted into the NdeI-KpnI sites of pAT28-LLO:1-35, yielding plasmid pAT28-LLO-d4. The resulting protein was named LLO-d4.

LLO-IL. A DNA fragment containing the promoter region of hly and the portion encoding domains d123 (residues 1 to 416) followed by a linker encoding the hexapeptide GGSGGS and an NdeI site was amplified by PCR using the primers $5^{\prime}$ GCTCTAGATCTCTTAAAGTGACTTTTATGTTGAGGCA-3' (primer 1) and 5'-GGCATATGGGATCCTCCGGATCCTCCATCTGTATAAGCTTTTGAAGTTGTTTCAATATATTCTGAG-3' (primer 2). The NdeI site was designed to be in-frame with the NdeI site present in construction pAT28-LLO-d4 (see above). The amplified fragment was first cloned into the pCR cloning vector. Then the XbaI-NdeI fragment encoding LLO-d123 was substituted for the XbaINdeI fragment of plasmid pAT28-LLO-d4. The sequence of the modified hinge region thus corresponds to the insertion of the nonapeptide GGSGGSHMTD flanked on both sides by the predicted original G417 linker.

LLO-IS. A DNA fragment containing the promoter region of bly and the portion encoding residues 1 to 414 of $\mathrm{d} 123$, followed by a NdeI restriction site was first amplified by PCR with the primers 5'-GCTCTAGATCTCTTAAAGTGACTTTTATGTTGAGGCA-3' (primer 1) and 5'-CCCATATGATAAGCTTTTGAAGTTGTTTCAATATA-3' (primer 2). The amplified fragment was first cloned into the pCR cloning vector. Then the XbaI-NdeI fragment encoding LLO-d123 was substituted for the XbaI-NdeI fragment of plasmid pAT28-LLO-d4. The resulting modified protein thus corresponds to the insertion of the dipeptide HM between residues 414 and 415 of LLO.

Simultaneous expression of LLO-d123 and LLO-d4. First, the gene encoding LLO-d123 was integrated into the chromosome of EGD $\Delta h l y$. For that, we used the integrative vector pAT113 (Trieu-Cuot et al., 1991; Autret et al., 2001). Integration of pAT113 in the chromosome of Gram-positive bacteria requires the presence of the transposon-encoded integrase in the recipient. Therefore EGD $\Delta$ hly was transformed with plasmid pAT145, carrying the transposon-encoded integrase Int-Tn. The BamHI-SalI fragment of plasmid pAT28-LLOd123 was cloned into the BamHI-SalI sites of vector pAT113 and the resulting plasmid (pAT113-LLO-d123) was transferred into EGD $\Delta h l y / \mathrm{pAT} 145$ by electroporation. One transformant, corresponding to a single chromosomal insertion (and showing normal growth capacities; not shown) was reserved. Finally, plasmid pAT28-LLO-d4, encoding LLO-d4, was introduced into this strain by electroporation.

\section{Protein preparation and analysis}

Protein preparation. The LLO proteins were prepared from supernatants of EGD $\Delta$ hly transformed with the different pAT2 8 derivatives. For each mutant, $25 \mathrm{ml}$ of an $8 \mathrm{~h}$ culture at $37^{\circ} \mathrm{C}$ in BHI-spectinomycin broth were added to $500 \mathrm{ml}$ 
RPMI 1640 minimal medium containing glucose (3\% final). The suspension was grown overnight with agitation at $37^{\circ} \mathrm{C}$ (under these conditions, the cultures corresponded to $\sim 2 \times 10^{8}$ bacteria $\mathrm{ml}^{-1}$ ). After centrifugation, cell-free supernatants were filtered through a $0.22 \mathrm{~mm}$ pore size Millipore filter. The filtered supernatants were first concentrated to $15 \mathrm{ml}$ by tangential flux through miniplate YM30 (Millipore) with a cut-off of $30 \mathrm{kDa}$ (except for LLO-d4 and LLOd123 + LLO-d4 expressed simultaneously, and the negative control EGD $\Delta h l y$, for which a cut-off of $10 \mathrm{kDa}$ was used). Supernatants were further concentrated to a final volume of 1 to $1.5 \mathrm{ml}$ by centrifugation through ultrafree Biomax units. The total protein concentration of the preparations, determined by the Bradford colorimetric method, was $\sim 1 \mathrm{mg} \mathrm{ml}^{-1}$.

Western-blot analysis. Ten microlitres of each concentrated supernatant were loaded per well onto SDS-13\% polyacrylamide gels. SDS-PAGE and Western-blot analyses were performed as described previously (Charbit et al., 2000) with polyclonal and monoclonal antibodies (see below).

Quantification. The amounts of LLO present in the concentrated supernatants were determined by dot-blot assays. Serial twofold dilutions of each preparation were coated onto nitrocellulose sheets and detection was carried out with monoclonal (mAb SE2) or polyclonal anti-LLO antibodies. Serial twofold dilutions of purified LLOwt were used as standards in the assay (starting from $1 \mu \mathrm{g}$ protein). The amounts of LLO detected in each spot were quantified by densitometry scanning of the nitrocellulose sheet using the NIH image software version 1.61 . They varied between $1 \%$ and $5 \%$ of total proteins. On this basis (i.e. 10 to $50 \mu \mathrm{g}$ LLO for $\sim 10^{11}$ bacteria), the number of molecules of LLO produced under our growth conditions corresponds to $10^{3}-$ $5 \times 10^{3}$ per bacterium, which is in agreement with previously reported values (Geoffroy et al., 1989; Villanueva et al., 1995). The final concentration of LLO in each protein preparation was finally adjusted to $0.5 \mu \mathrm{g} \mu \mathrm{l}^{-1}$.

Antibodies. A polyclonal anti-LLO serum, raised in rabbits against denatured LLO (Geoffroy et al., 1989), was used in Western blots and dot blots at a final dilution of $1 / 1000$ and in immunofluorescence at a final dilution of 1/500. A monoclonal anti-LLO antibody, mAb SE2 (kindly provided by Dr A. J. Ainsworth, Veterinary Medical Research, College of Veterinary Medicine, Mississippi State University, MS, USA), raised in mice after injection of concentrated L. monocytogenes extracellular proteins (Erdenlig et al., 1999), was used in Western blots and dot blots at a final dilution of $1 / 1000$ and in immunofluorescence at a final dilution of $1 / 100$.

A monoclonal anti-pneumolysin antibody, mAb PLY-5 (kindly provided by Dr J. R. de los Toyos, Area de Immunologia, Facultad de Medicina, Universidad de Oviedo, Spain), was used in the membrane-binding assay to inhibit binding of LLO-d4 to erythrocyte membranes. This mAb was previously shown to recognize a peptide within the conserved undecapeptide at the tip of the $\mathrm{d} 4$ domains of thiol-activated cytolysins, including LLO (Jacobs et al., 1999).

Immunoprecipitation. Concentrated supernatants were first incubated with mAb SE2 $(25 \mu \mathrm{l}$ antibody in $250 \mu \mathrm{l}$ final $)$ and then immunoprecipitated with $50 \mu \mathrm{l}$ protein A-agarose $(25 \mu \mathrm{l}$ bed volume from a suspension at $3 \mathrm{mg} \mathrm{m}^{-1}$; Boehringer Mannheim). For LLO-d123 + LLO-d4 expressed simultaneously (denoted $123+4$ ) or separately (denoted $123+4 \mathrm{rec}$ ), $2 \mu \mathrm{g}$ LLO were used ( $1 \mu \mathrm{g}$ LLO-d123 + $1 \mu \mathrm{g}$ LLO-d4). Protein preparations of either LLO-d123 or LLO-d4 alone were used as negative controls $(1 \mu \mathrm{g})$. After electrophoresis, immunoprecipitated proteins were transferred onto nitrocellulose membranes. The LLO proteins were finally revealed using the rabbit polyclonal anti-LLO serum (1/1000 final). The assay was repeated twice.

Haemolysis. Haemolytic phenotypes were visualized by spreading bacteria onto horse-blood agar plates (bioMérieux). Haemolytic activity was measured at pH 6.6 as described previously (Geoffroy et al., 1989; Portnoy et al., 1988). Serial twofold dilutions of filtered supernatants from BHI-grown bacteria (starting from $20 \mu \mathrm{l}$ supernatant) were incubated with $50 \mu \mathrm{l}$ horse red blood cells (HRBC) at $\mathrm{OD}_{541} 0 \cdot 2$, in a final volume of $100 \mu \mathrm{l}$. The haemolytic activity was estimated as the reciprocal of the dilution giving $50 \%$ haemolysis.

The haemolytic activity of the concentrated supernatants was also measured at $\mathrm{pH} 6 \cdot 6$. Serial twofold dilutions of each LLO preparation, starting from $1 \mu \mathrm{g}$ LLO in the first well, were tested at different concentrations of $\operatorname{HRBC}\left(\mathrm{OD}_{541} 0 \cdot 2,0 \cdot 1\right.$ or $0 \cdot 05)$.

\section{Membrane-binding assays}

Binding to erythrocyte membranes. Horse erythrocytes $(50 \mathrm{ml})$ were lysed by sonication (on ice) with $20 \mathrm{mM} \mathrm{MgCl}_{2}$. After sonication, unbroken cells were removed by low-speed centrifugation $(1000 \mathrm{~g}$ for $20 \mathrm{~min}$ ). The supernatant was dialysed overnight at $4{ }^{\circ} \mathrm{C}$ in PBS $/ 20 \mathrm{mM} \mathrm{MgCl}$. Dialysed membranes were concentrated by ultracentrifugation at $23500 \mathrm{~g}$ for $1 \mathrm{~h}$ and finally resuspended in $\mathrm{PBS} / 20 \mathrm{mM} \mathrm{MgCl}_{2}$ at a concentration of $0.4 \mathrm{~g} \mathrm{l}^{-1}$ (final).

Each concentrated supernatant, containing $5 \mu \mathrm{g}$ LLO, was incubated with $80 \mu \mathrm{g}$ erythrocyte membranes (unless otherwise stated) in a final volume of $1 \mathrm{ml}$ for $30 \mathrm{~min}$ at room temperature. The mixture was then centrifuged at $23500 \mathrm{~g}$ for $1 \mathrm{~h}$ at $4{ }^{\circ} \mathrm{C}$. The pellets containing the membrane were solubilized in SDS-PAGE loading buffer. Toxins from the supernatant were recovered by TCA precipitation. Each fraction (pellet or supernatant) was finally concentrated by centrifugation at $23500 \mathrm{~g}$ for $1 \mathrm{~h}$ at $4{ }^{\circ} \mathrm{C}$. After electrophoresis, proteins were transferred onto nitrocellulose membranes. The LLO proteins were finally revealed using the rabbit polyclonal anti-LLO serum (1/1000 final). The percentage of LLO in the bound (pellet) and unbound (supernatant) fractions was determined by densitometry scanning of the nitrocellulose sheet.

Inhibition of LLO-d4 binding was performed after a preincubation with $1 \mu \mathrm{l} \mathrm{mAb} \mathrm{PLY}-5$ for $30 \mathrm{~min}$ at $37^{\circ} \mathrm{C}(5 \mu \mathrm{g}$ LLO in a final volume of $100 \mu \mathrm{l})$.

Binding to intact cells. We used the epithelial cell line HeclB, a human endometrial adenocarcinoma cell line (obtained from the American Type Culture Collection, Manassas, VA, USA). Cells were cultured in Dulbecco's Modified Eagle's Medium (DMEM; Gibco), containing 10\% foetal bovine serum at $37^{\circ} \mathrm{C}$ under $5 \% \mathrm{CO}_{2}$. Cells were seeded at $8 \times 10^{4}$ cells $\mathrm{cm}^{-2}$ onto $12 \mathrm{~mm}$ diameter glass coverslips in 24-well plates. Monolayers were used $24 \mathrm{~h}$ after seeding.

Purified LLO-d123 and LLO-d4 were used in this assay. Binding of LLO-d123 and LLO-d4 to the surface of intact cells was detected by confocal microscopy, using either anti-LLO $\mathrm{mAb}$ SE2 for LLO-d123 or the polyclonal serum for LLO-d4. HeclB cells were incubated with $1 \mu \mathrm{g}$ LLO d123 or LLO-d4 for $30 \mathrm{~min}$ at $37^{\circ} \mathrm{C}$. After three washes with PBS, cells were fixed with $3 \%(\mathrm{w} / \mathrm{v}$ ) paraformaldehyde (in PBS) for $30 \mathrm{~min}$ and washed three times with PBS. Cells were then processed for 
fluorescence labelling. Cells were incubated sequentially with either mAb SE2 diluted 1/100 and then with CY3-labelled anti-mouse IgG (Jackson Immunoresearch Laboratories) diluted $1 / 200$, or with the polyclonal serum diluted $1 / 500$ and then with anti-rabbit IgG coupled to Alexa 546 diluted 1/200. Incubations were carried out for $30 \mathrm{~min}$ at room temperature and followed by three washings in PBS. Coverslips were mounted on slides and examined by fluorescence microscopy with a Leica TCS4D confocal laser scanning microscope. Each assay was repeated several times.

Sequence searches, alignments and graphics. Similarity searches were done via the internet with BLAST software (Altschul et al., 1997). The theoretical 3D model of LLO folding was produced using the Automated Comparative Protein Modelling Server (available athttp://www.expasy.ch/ swissmod/SWISS-MODEL.html) (Guex et al., 1999 and references therein) and the RasMac Program (Version RasMol v2.6, 1994. Available from Roger Sayle, Biomolecular Structure, Glaxo Research and Development, Greenford, UK), using the X-ray coordinates of PFO.

\section{RESULTS}

\section{Sequence analyses and structural predictions}

LLO shares significant similarities with the other members of the cytolysin family. However, sequence alignments reveal that conservation is significantly higher in the $\mathrm{d} 4$ domain region (with $62-73 \%$ identity) than in the proximal $2 / 3$ portion (ranging from $38 \%$ to $71 \%$ identity; not shown). In particular, the identity between LLO and PFO polypeptides is $64 \%$ in the $\mathrm{d} 4$ domain (residues 416 to 529 in the LLO molecule) while it is only $38 \%$ in the $\mathrm{d} 123$ domains (LLO residues 1 to 415). Sequence similarities between LLO and PFO allowed the elaboration of the theoretical 3D model of LLO folding shown in Fig. 1 (a). In the crystal structure of PFO (Rossjohn et al., 1997), d4 and d2, which are connected together through a glycine linker, are in partial contact via one salt link and several hydrogen bonds centred about an aromatic cluster. The glycine linker (G417) and the aromatic cluster (Y98, Y414 and Y440) are conserved in the LLO sequence (LLO preprotein numbering). Remarkably, in the predicted structure of LLO, four aromatic residues (the aromatic cluster and Y92) are located at the interface of d123 and $\mathrm{d} 4$ with their side chains perpendicular to the plane of the molecule (not shown).

Using this theoretical 3D model of LLO folding, we constructed four LLO mutants (Fig. 1b) - two truncated proteins and two linker insertion mutants - and expressed them in EGD $\Delta h l y$. We have focused here mainly on the capacity of the truncated proteins to bind to eukaryotic membranes and to reassemble simultaneously to form an active molecule.

\section{Expression of the LLO mutant proteins in L. monocytogenes}

Two truncated LLO proteins corresponding to domains 1, 2 and 3 (LLO-d123) or domain 4 (LLO-d4), and two linker-insertion mutants corresponding to in-frame in- (a)

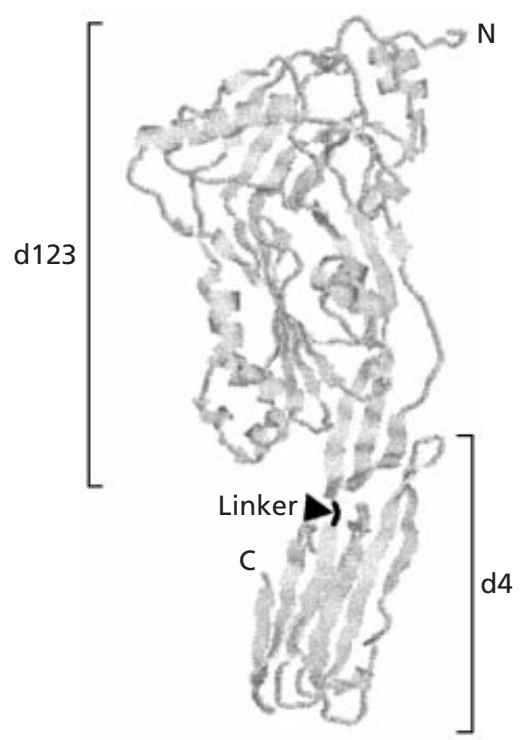

(b)

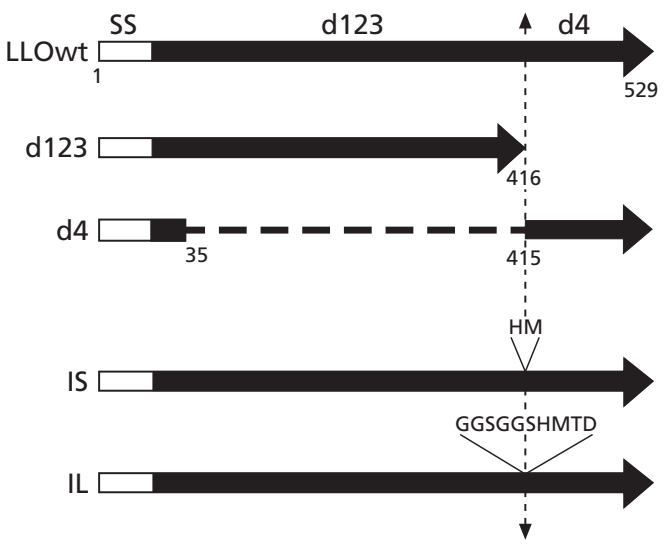

Fig. 1. Structural predictions of LLO. (a) Theoretical 3D model of LLO folding. The position of the linker is indicated by an arrowhead. (b) Schematic representation of the mutant proteins. All the constructions were carried on pAT28-derived plasmids under phly control. Domain designations are provided above the linear depictions. The region of the signal sequence (denoted SS) is drawn in white. The mature portion of LLO is drawn in black. The residues noted below indicate the first and the last residue of the pre-protein. A dotted vertical arrow indicates the boundaries between $\mathrm{d} 123$ and $d 4$. The residues of the inserted linkers are indicated above each linear depiction.

sertion of two (LLO-IS) or ten residues (LLO-lL) at amino acid site 416, were constructed (Fig. 1b). The recombinant proteins were expressed in EGD $\Delta$ hly from plasmid-borne genes. EGD $\Delta h l y$ expressing LLOwt was used as a positive control (Dubail et al., 2000).

The four LLO mutant proteins were efficiently secreted into the culture supernatant of L. monocytogenes. As shown in the Western blot of Fig. 2 (a), LLO-d123 and LLO-d4 were detected by the polyclonal antiLLO antibody in concentrated culture supernatants. 
(a)

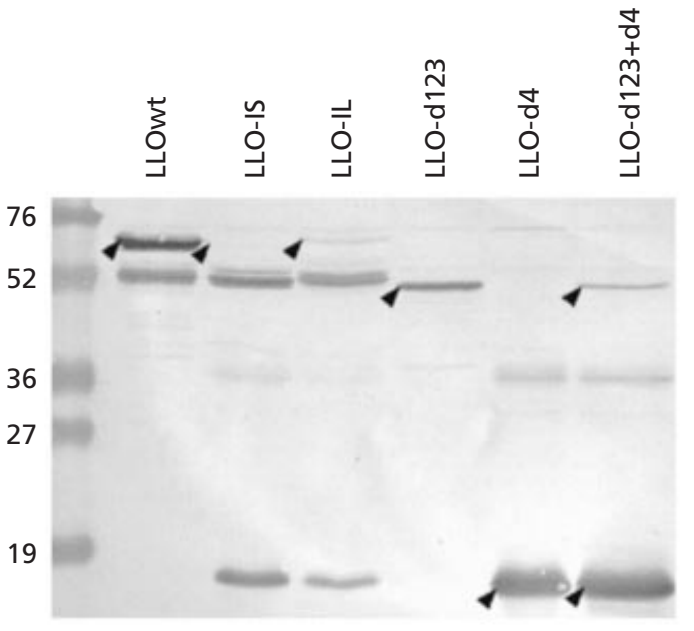

(b)

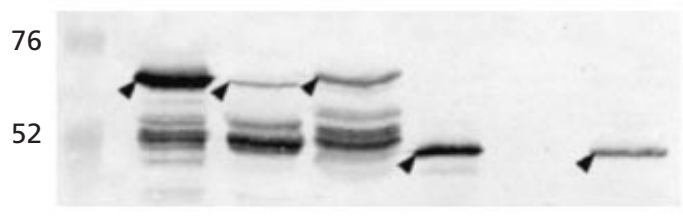

Fig. 2. Immunodetection of the LLO mutants. LLO-d123 $+\mathrm{d} 4$ (last lane) is the supernatant of $\operatorname{EGD} \Delta h l y$ expressing simultaneously LLO-d123 and LLO-d4. Ten microlitres of concentrated culture supernatant were loaded onto an SDS-13\% polyacrylamide gel. Proteins were transferred electrophoretically onto nitrocellulose and membranes were incubated with either anti-LLO polyclonal antibody (a) or antiLLO monoclonal antibody SE2 (b). The apparent molecular masses of the marker are indicated on the left (in kDa). Arrowheads indicate the bands corresponding to the migration of intact proteins.

Strikingly, for the two linker mutants (LLO-IS and LLOIL), two major degradation species were recognized. The apparent migration of the two species detected ( $\sim 50$ and $15 \mathrm{kDa}$, respectively) is compatible with a cleavage of the mutant proteins in the vicinity of the linker insertion that would generate peptides corresponding to $\mathrm{d} 123$ and $\mathrm{d} 4$ domains. The monoclonal anti-LLO antibody (mAb SE2) recognized LLOwt and LLO-d123 but failed to recognize LLO-d4, indicating that its recognition site lies within the proximal $2 / 3$ portion of the molecule (Fig. 2b). mAb SE2 could also recognize the mature form of the two linker-insertion mutants as well as a species of $\sim 50 \mathrm{kDa}$. The fact that the $15 \mathrm{kDa}$ species, detected with the polyclonal serum, was not detected by the mAb (not shown) further supports the idea that this peptide might correspond to the $\mathrm{d} 4$ domain of LLO.

The amounts of LLO secreted into the supernatant of L. monocytogenes were quantified by dot-blot assays on concentrated culture supernatants, using the monoclonal and polyclonal anti-LLO antibodies (see Methods for details). The amounts of LLO-d123, LLO-IS and LLO-IL produced were comparable and corresponded
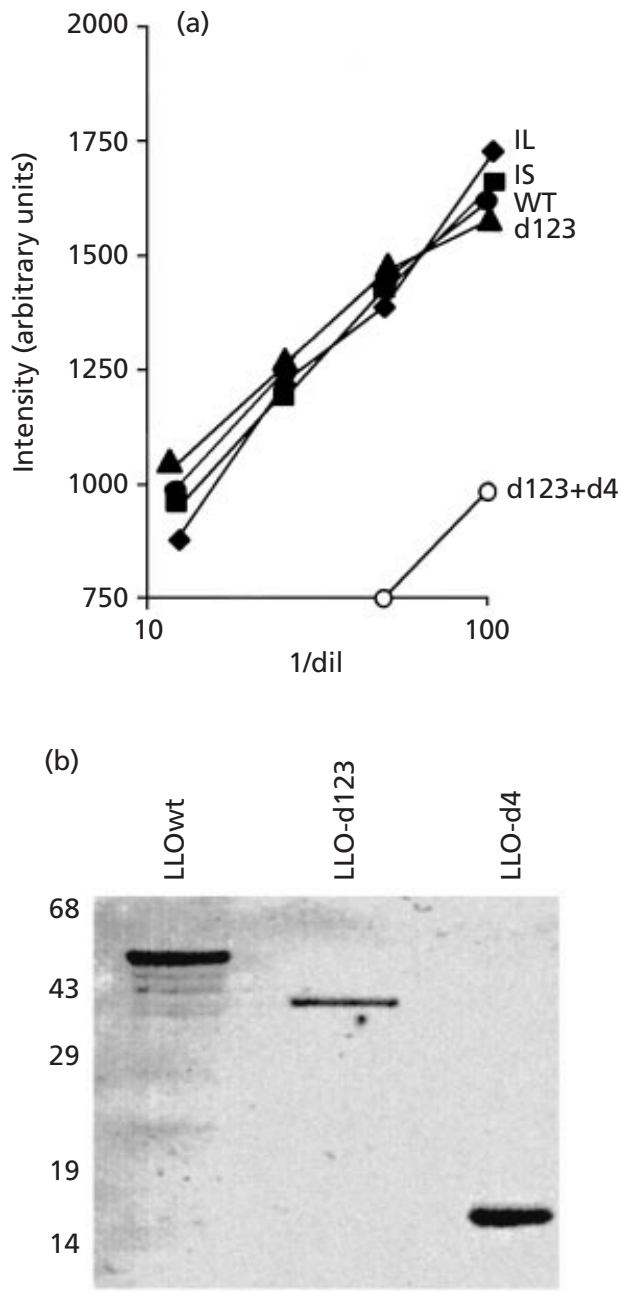

Fig. 3. Quantification of LLO. (a) The amounts of LLO proteins expressed by EGD $\Delta h l y$ were determined in the concentrated culture supernatants by dot-blot assays. Serial twofold dilutions of each preparation (starting from $50 \mu \mathrm{l}$ extract for LLO-d123, LLO-IS and LLO-IL, and from $12.5 \mu \mathrm{l}$ extract for LLOwt) were spotted onto nitrocellulose. The coated proteins were detected with mAb SE2 (final dilution of 1:1000). The assay was revealed with anti-mouse immunoglobulin-peroxidase conjugate (final dilution of $1: 1000$ ). 1/dil shown on the abscissa is the reciprocal of the dilution of concentrated supernatant coated multiplied by 100 (i.e. 100 is the non-diluted sample). The intensity is shown on the ordinate as arbitrary units recorded by densitometric analysis using the $\mathrm{NIH}$ image software version 1.61. (b) Coomassie blue stained gel loaded with purified LLO, LLO-d123 and LLO-d4. The apparent molecular masses of the marker are indicated on the left (in kDa).

to approximately one quarter that of LLOwt (Fig. 3). Similar results were obtained with the polyclonal antiLLO serum (not shown). In the preparation corresponding to the simultaneous expression of LLO-d4 and LLO-d123, the amounts of chromosomally encoded LLO-d123 appeared to be $\sim 8$-fold lower than that of the plasmid-encoded LLO-d123. The amounts of LLO-d4 in the two preparations containing LLO-d4, expressed alone or with LLO-d123, were similar to that 
of LLOwt. Since mAb SE2 did not recognize LLO-d4 (see Results), detection was performed with the polyclonal serum.

The concentration of LLO in the different protein preparations was finally adjusted to $50 \mu \mathrm{g} \mathrm{ml}^{-1}$ for all the subsequent assays. The haemolytic activity of the four LLO mutants was assayed on horse blood agar plates (not shown) and quantified by titration on horse erythrocytes (see Methods for details). None of them showed any detectable haemolytic activity.

\section{Binding of LLO-d123 and LLO-d4 to eukaryotic membranes}

Wild-type LLO is known to bind to cholesterolcontaining membranes (Jacobs et al., 1998). The ability of the mutant proteins to bind to eukaryotic cell membranes was tested on erythrocyte membranes and on intact eukaryotic cells.

Binding to erythrocyte membranes. This was assayed essentially as described by Jacobs et al. (1998) on concentrated supernatants. Each protein preparation, containing $5 \mu \mathrm{g}$ LLO, was incubated with erythrocyte membranes $\left(80 \mu \mathrm{g} \mathrm{ml}^{-1}\right.$ final). After $30 \mathrm{~min}$ incubation at room temperature, the membranes were collected by centrifugation and subjected to SDS-PAGE (see Methods). The LLO fraction bound to the membranes was revealed by Western blotting with anti-LLO polyclonal serum. As shown in Fig. 4(a), the four LLO mutant proteins (including LLO-d123 and LLO-d4 expressed simultaneously) bound to erythrocyte membranes. Strikingly, with both linker-insertion mutants, the two major proteolytic species were still detected. For LLO-d123, $\sim 50 \%$ of the protein was detected in the membrane fraction and $50 \%$ in the supernatant : a value comparable to that obtained with LLOwt. In contrast, for LLO-d4, the bound fraction corresponded to only $15-20 \%$ (as determined by densitometry scanning of the nitrocellulose sheet using the NIH image software version 1.61).

The amounts of LLO-d123 and LLO-d4 bound to the membranes were also compared at different concentrations of membranes. Identical amounts of each mutant protein $(5 \mu \mathrm{g})$ were incubated with 40,80 or $160 \mu \mathrm{g}$ erythrocyte membranes. With both mutants, the intensity of the band detected in the membrane-bound fraction was significantly higher with $80 \mu \mathrm{g}$ than with $160 \mu \mathrm{g}$ membranes (Fig. 4b). The optimal concentration of $80 \mu \mathrm{g}$ membranes per assay was then used to compare the percentage of LLO-d123 bound to the membranes with lower amounts of protein (see Methods for details). The percentage of bound LLO-d123 rose from $53 \%$ with $1 \mu \mathrm{g}$ LLO-d 123 to $75 \%$ with $0 \cdot 25 \mu \mathrm{g}$. Thus, within the limits of detection of the assay (from $\sim 0.18 \mu \mathrm{g}$ to $0.5 \mu \mathrm{g}$ bound LLO), binding increased linearly.

Two sets of controls were performed to check the specificity of the binding. i) The assay was carried out without membranes. Under these conditions, the two truncated and the two-linker insertion mutant proteins
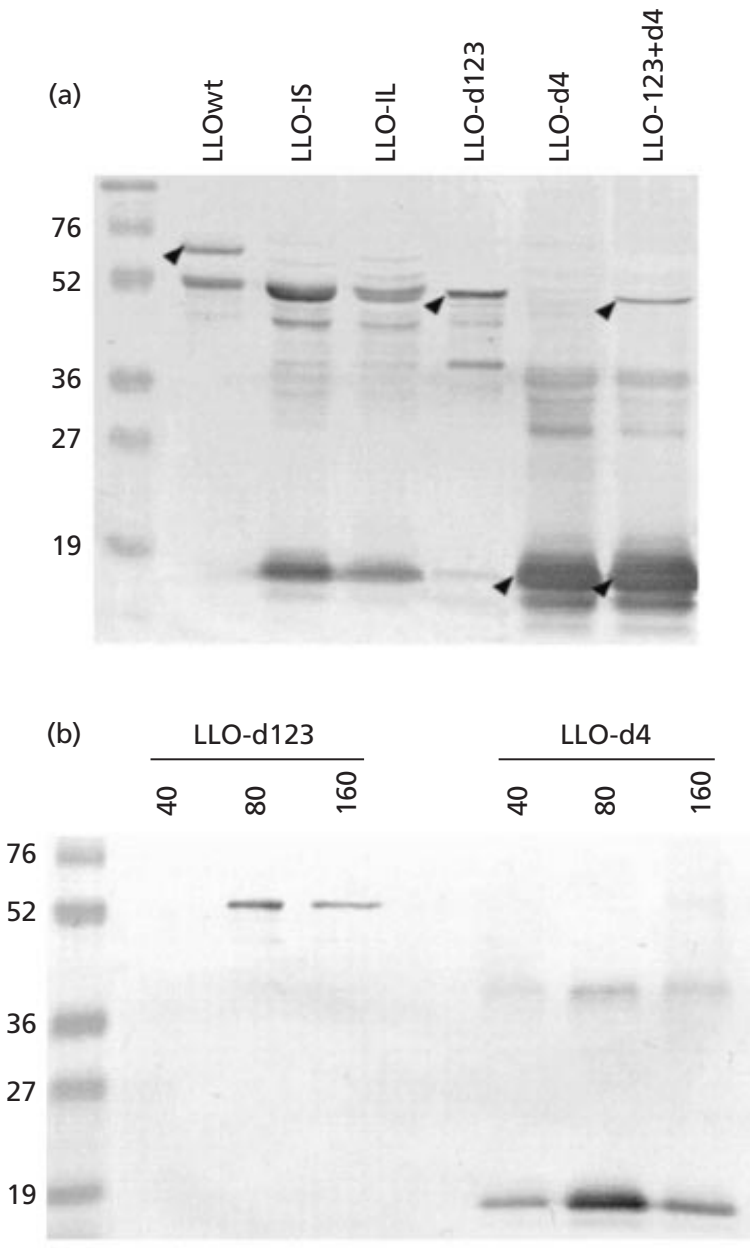

(c)

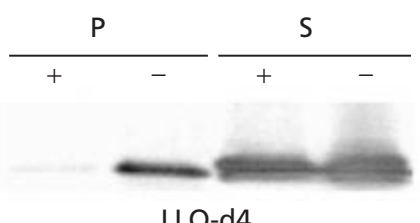

Fig. 4. Binding of the LLO mutant proteins to erythrocyte membranes. HRBC membranes were incubated with identical amounts of the different LLO preparations. The pellet fractions (membrane-bound proteins) were solubilized in the same final volume of loading buffer and applied onto an SDS-13\% polyacrylamide gel. Proteins were transferred electrophoretically onto nitrocellulose. The immunoblots were developed using anti-LLO polyclonal antibody (at a final dilution of 1/1000). (a) Membrane fractions of HRBC incubated with the different LLO mutants. Arrowheads indicate the bands corresponding to non-degraded proteins. The molecular masses (in $\mathrm{kDa}$ ) of the marker are indicated on the left. Five micrograms of LLO and $80 \mu \mathrm{g}$ membranes were used per assay. (b) Membrane fractions of HRBC membranes incubated with LLO-d123 or LLO-d4. For each preparation, $5 \mu \mathrm{g}$ LLO were incubated with increasing amounts of HRBC membranes $(40,80$ and $160 \mu \mathrm{g}$ ). (c) Membrane (P) and supernatant (S) fractions of HRBC membranes incubated with LLO-d4. + indicates a $30 \mathrm{~min}$ preincubation with mAb PLY-5; - indicates no preincubation. Five micrograms of LLO-d4 and $80 \mu \mathrm{g}$ membranes were used per assay. 

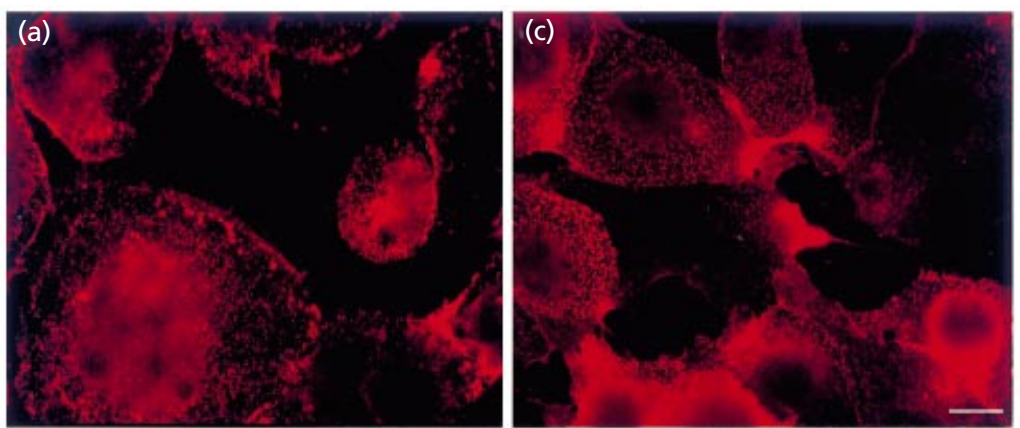

(b)

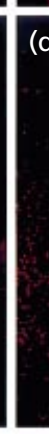

Fig. 5. Binding of LLO-d 123 and LLO-d 4 to epithelial cells. Binding of purified LLO-d123 and LLO-d4 to intact cells was revealed with monoclonal (LLO-d123) or polyclonal (LLO-d4) anti-LLO antibodies, followed by incubation with either CY3-labelled antimouse IgG (diluted 1/200) or anti-rabbit IgG conjugated with Alexa 546 (diluted 1/200), respectively. (a) Epithelial cells incubated with $1 \mu \mathrm{g}$ LLO-d123. (b) As a negative control, epithelial cells were incubated with a supernatant from EGD $\Delta h l y$ alone. In both cases, mAb SE2 was used at a final dilution of 1/100. (c) Epithelial cells incubated with $1 \mu \mathrm{g}$ LLO-d4. (d) Epithelial cells not incubated. In both cases, anti-LLO polyclonal serum was used at a final dilution of 1/500. were essentially found in the supernatant. ii) The assay was performed with a heterologous protein, i.e. the maltose-binding protein (MBP) of Escherichia coli K-12 (Betton \& Hofnung, 1994). MBP was essentially found in the supernatant (not shown). These two assays demonstrated that the presence of the LLO mutant proteins in the membrane fractions (pellets) was not due to the formation of non-specific protein aggregates and confirmed that the binding was specific.

Preincubation of LLO-d4 with mAb PLY-5 (directed against the $\mathrm{d} 4$ domain) almost totally inhibited the binding of LLO-d4 to erythrocyte membranes (Fig. 4c). This result is in agreement with previous data suggesting that binding of LLO involved directly its conserved motif, located in the $\mathrm{d} 4$ domain. However, it cannot be excluded that inhibition might be due to steric hindrance by the $\mathrm{mAb}$ and that membrane binding occurs via another portion of the LLO-d4 polypeptide.

Binding to intact cells. Binding of LLO-d123 and LLO-d4 to the surface of intact eukaryotic cells was then evaluated by confocal microscopy on human epithelial cells, using mAb SE2 (directed against this proximal portion of the molecule). One microgram of purified protein was used (Fig. 3b). As shown in Fig. 5(a), a strong and uniformly distributed binding of LLO-d123 protein was observed on all the cells. As a negative control, cells were preincubated with a supernatant from EGD $\Delta h l y$ alone and we did not observe any nonspecific binding of the monoclonal antibody to cell membranes (Fig. 5b). Under the conditions of the assay, using mAb SE2, only a very weak labelling was observed when cells were preincubated with the LLO linker derivatives (not shown). The binding of LLO-d4 to intact cells was tested using the polyclonal anti-LLO serum. As shown in Fig. 5 (c), binding of LLO-d4 was also uniformly distributed on the surface of all the cells. As a negative control, the polyclonal serum was tested on cells that were not preincubated with LLO : only a very weak background signal was recorded under these conditions (Fig. 5d).

\section{Functional assembly of domains d123 and d4 of LLO}

We tested whether the simultaneous production of the two polypeptides by the same strain allowed reassembling of the two polypeptides i) physically, by immunoprecipitation and ii) functionally, by measuring the haemolytic activity of concentrated culture supernatants. The DNA gene fragment encoding the first 416 residues of LLO (LLO-d123) was integrated at random in the chromosome of EGD $\Delta h l y$ using an integrative vector (Autret et al., 2001). One chromosomal insertion that did not affect bacterial growth was chosen and the resulting strain was transformed with the plasmid encoding LLO-d4. In this strain, each recombinant gene is under phly promoter control (see Methods). The two polypeptides were efficiently secreted in the culture supernatant, as shown by Western-blot analysis with anti-LLO polyclonal serum (Fig. 3a).

Co-immunoprecipitation of LLO-d4 with LLO-d123. A concentrated supernatant from EGD $\Delta h l y$ expressing simultaneously the two polypeptides was incubated with mAb SE2 (specific for LLO-d123). The antigen-antibody complexes were immunoprecipitated with protein Aagarose and subjected to SDS-PAGE. After electrophoresis, proteins were transferred onto nitrocellulose membranes and the LLO proteins were revealed with rabbit polyclonal anti-LLO (recognizing both LLO-d123 and LLO-d4). As expected, LLO-d123 was efficiently immunoprecipitated by $\mathrm{mAb}$ SE2 and revealed by the polyclonal serum (Fig. 6a). There was not any 
(a)

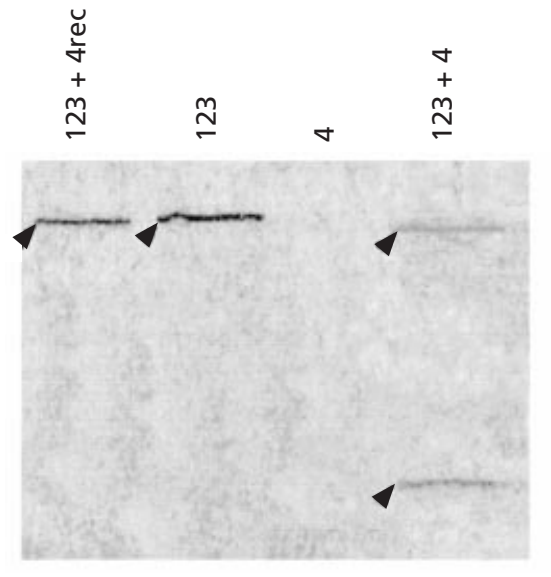

(b)

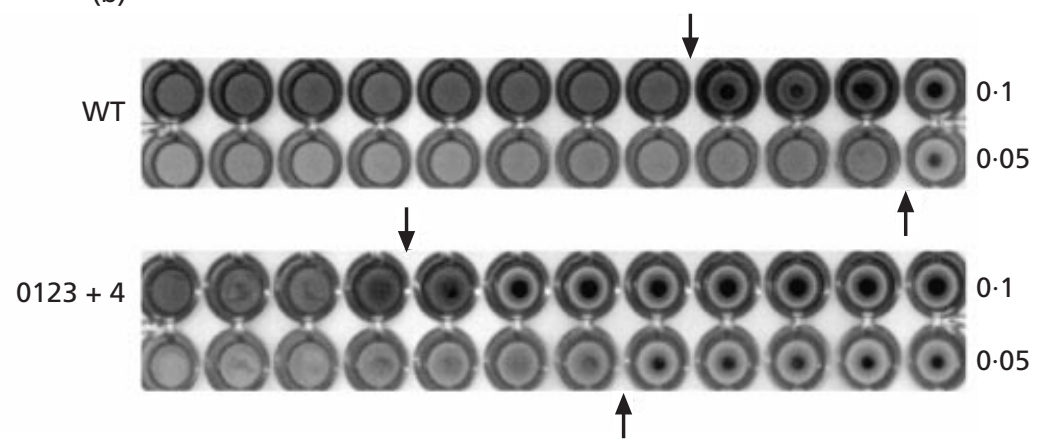

Fig. 6. Functional assembly of LLO-d123 and LLO-d4. (a) Immunoprecipitation. LLO proteins were incubated for $1 \mathrm{~h}$ at $37^{\circ} \mathrm{C}$ with mAb SE2. The LLO-d123-mAb complexes were then immunoprecipitated with protein A-agarose. The mixture was submitted to SDS-PAGE. Immunoprecipitated proteins were transferred electrophoretically onto nitrocellulose and incubated with anti-LLO polyclonal serum. $123+4 \mathrm{rec}$ is LLO-d123 and LLO-d4 produced separately; 123 is LLO-d123; 4 is LLO-d4; $123+4$ is LLO-d123 and LLO-d4 produced simultaneously. Arrowheads indicate the bands corresponding to LLOd123 and LLO-d4. (b) Haemolytic activity of LLO-d123+LLO-d4. HRBC were incubated with serial twofold dilutions of concentrated protein supernatant (starting from $1 \mu \mathrm{g}$ LLO in the first well). Cytolysis of erythrocytes is visualized by a uniform grey colour indicating haemoglobin release. A dark spot at the bottom of the well is sedimented intact erythrocytes. The arrows show the highest dilution of protein preparation yielding haemolysis. The two $O D_{541}$ values of HRBC used are indicated on the right (0.1 and 0.05). WT, LLOwt; $123+4$, LLO-d123 and LLO-d4 expressed simultaneously.

non-specific reaction with LLO-d4. In the concentrated supernatant containing both polypeptides $(123+4$, simultaneous expression), LLO-d4 was co-immunoprecipitated with LLO-d123, demonstrating that the proteins were able to interact physically. In contrast, when LLO-d123 and LLO-d4 produced separately were mixed $(123+4 \mathrm{rec})$, only LLO-d123 was immunodetected.

Haemolysis. The haemolytic activity of the concentrated supernatant $\mathrm{d} 123+4$ was tested at $\mathrm{pH} 6.6$ on horse erythrocytes at $\mathrm{OD}_{541} 0 \cdot 1$ and $0 \cdot 05$ (Fig. 6b), and compared to that of a concentrated supernatant containing LLOwt (see Methods). Under these conditions, the activity in the preparation $\mathrm{d} 123+4$ was $\sim 10$-fold lower than of the wild-type protein. The haemolytic activity of the preparation was completely inhibited by a preincubation with cholesterol (not shown), like the wild-type LLO protein (Lety et al., 2001 and references therein). Strikingly, under the same conditions, LLO$\mathrm{d} 123$ and LLO-d4 produced separately and mixed before incubation produced no detectable haemolytic activity. We also tested whether $\mathrm{d} 123$ and $\mathrm{d} 4$ produced separately and mixed together could recover a cytolytic activity after denaturation in $8 \mathrm{M}$ urea and renaturation by dialysis for $24 \mathrm{~h}$ at $4{ }^{\circ} \mathrm{C}$ in $50 \mathrm{mM}$ Tris/HCl, pH 7.5. This treatment failed to restore any detectable haemolytic activity.

These data, which demonstrated that LLO-d123 and LLO-d4 proteins could reassemble to form a haemolytically active LLO when expressed simultaneously, suggest that functional assembly of the two domains might occur during, or immediately after, the cosecretion of the two proteins.

\section{DISCUSSION}

We used a theoretical 3D model of LLO folding to design two truncated and two linker-insertion mutants. The data presented here showed that i) the two truncated proteins LLO-d123 and LLO-d4, when secreted simultaneously by L. monocytogenes, could reassemble to form a haemolytically active molecule, and ii) minor modifications in the region connecting $\mathrm{d} 4$ to the rest of the molecule drastically altered protein stability and hence activity.

\section{LLO-d123 and LLO-d4 bind to cell membranes and can reassemble to form an active molecule}

Earlier studies have shown that a C-terminal proteolytic fragment of PFO (residues 304-500) could bind erythrocytes like intact toxin (Tweten et al., 1991), and biophysical studies clearly established that domain 4 of PFO interacted with the bilayer (Nakamura et al., 1998). Further biophysical analyses revealed that each PFO monomer contained a second region (domain 3), involved in pore formation (Shatursky et al., 1999; Shepard et al., 1998). A structural linkage between domains 3 and 4 of PFO was very recently demonstrated (Heuck et al., 2000). That study showed that the two domains interacted sequentially with cholesterol-containing membranes: domain 4 interacted first, eliciting a conformational change in the proximal part of the molecule, allowing subsequent insertion of domain 3.

In agreement with these data, the present work revealed that LLO-d4 and LLO-d123, expressed individually, could bind to erythrocyte membranes and to intact eukaryotic cells. The binding capacity of the LLO-d123 alone might be due to a direct accessibility of the 
membrane-binding domain at the surface of the molecule. Further analyses will be required to determine the exact mode of interaction of this truncated protein with the membrane and of its oligomeric state in solution and upon binding to cell membranes.

LLO-d123 and LLO-d4, when secreted simultaneously by the same bacterium, could reassemble to form a haemolytically active molecule. Physical interactions between the two portions of LLO were confirmed by showing that the $\mathrm{d} 4$ domain could be immunoprecipitated with an antibody specifically directed against d123.

We also tested whether the expression of LLO-d123 could interfere with the activity of the chromosomally encoded wild-type protein (negative dominance). For that, we transformed EGDwt with the multicopy plasmid pAT28-LLO-d123 encoding LLO-d123. The haemolytic activity recorded in the culture supernatant of the recombinant strain was identical to that of the wild-type strain, indicating that the expression of the truncated polypeptide did not prevent pore formation (not shown).

Most of the mutations in LLO (or in PFO) affecting haemolytic activity identified so far were found in the $\mathrm{d} 4$ domain, within or in close vicinity to the conserved undecapeptide thought to be involved in cell binding (Michel et al., 1990; Jones et al., 1996). We have shown here that modifications in the region connecting d123 and $\mathrm{d} 4$ were also critical for the haemolytic activity of LLO, by increasing the susceptibility of the protein to proteolytic degradation. These data are in agreement with the currently accepted notion that $\mathrm{d} 123$ and $\mathrm{d} 4$ interact together to form an active cytolysin molecule (Palmer et al., 1998; Shepard et al., 1998, 2000; Ghani et al., 1999; Heuck et al., 2000). One hypothesis would be that the hinge region might be involved in this interaction. Biochemical and biophysical analyses of the truncated proteins should provide interesting information on the ability of the LLO protein domains to fold autonomously.

Finally, these data showed that the hinge region of LLO is not a 'permissive' region of the molecule. In this respect, it is worth mentioning that a region close to the $\mathrm{N}$ terminus of LLO was very recently found to be critical for bacterial virulence (Decatur \& Portnoy, 2000; Lety et al., 2001). This region is involved in phagosomal escape and might also regulate the intra-cytosolic half life of LLO. Strikingly, this proximal region of LLO allowed local sequence alterations without any detectable effect on protein secretion and haemolytic activity. At this stage, it is tempting to speculate that the hinge region between $\mathrm{d} 123$ and $\mathrm{d} 4$ of LLO could also be a target of proteolytic degradation in infected cells.

\section{ACKNOWLEDGEMENTS}

We thank Dr A. J. Ainsworth for providing the anti-LLO monoclonal antibody SE2, Dr J. R. de los Toyos for providing the anti-PLO monoclonal antibody PLY-5 and Dr C. Tinsley for critical reading of the manuscript. This work was supported by CNRS, INSERM and University Paris V and the EEC (BMH-4 CT 960659).

\section{REFERENCES}

Alouf, J. E. (2000). Cholesterol-binding cytolytic protein toxins. Int J Med Microbiol 290, 351-356.

Altschul, S. F., Madden, T. L., Schaffer, A. A., Zhang, J., Zhang, Z., Miller, W. \& Lipman, D. J. (1997). Gapped BLAST and PSI-BLAST : a new generation of protein database search programs. Nucleic Acids Res 25, 3389-3402.

Autret, N., Dubail, I., Trieu-Cuot, P., Berche, P. \& Charbit, A. (2001). Identification of new genes involved in the virulence of Listeria monocytogenes by signature-tagged transposon mutagenesis. Infect Immun 69, 2054-2065.

Bayley, H. (1997). Toxin structure: part of a hole? Curr Biol 7, R763-R767.

Berche, P., Gaillard, J. L. \& Sansonetti, P. J. (1987). Intracellular growth of Listeria monocytogenes as a prerequisite for in vivo induction of $\mathrm{T}$ cell-mediated immunity. J Immunol 138, 2266-2271.

Betton, J. M. \& Hofnung, M. (1994). In vivo assembly of active maltose binding protein from independently exported protein fragments. EMBO J 13, 1226-1234.

Bibi, E. \& Kaback, H. R. (1990). In vivo expression of the lacY gene in two segments leads to functional lac permease. Proc Natl Acad Sci U S A 87, 4325-4329.

Chakraborty, T., Leimeister-Wachter, M., Domann, E., Hartl, M., Goebel, W., Nichterlein, T. \& Notermans, S. (1992). Coordinate regulation of virulence genes in Listeria monocytogenes requires the product of the prfA gene. J Bacteriol 174, 568-574.

Charbit, A., Andersen, C., Wang, J., Schiffler, B., Michel, V., Benz, R. \& Hofnung, M. (2000). In vivo and in vitro studies of transmembrane beta-strand deletion, insertion or substitution mutants of the Escherichia coli K-12 maltoporin. Mol Microbiol 35, 777-790.

Decatur, A. L. \& Portnoy, D. A. (2000). A PEST-like sequence in listeriolysin $\mathrm{O}$ essential for Listeria monocytogenes pathogenicity. Science 290, 992-995.

Diep, D. B., Lawrence, T. S., Ausio, J., Howard, S. P. \& Buckley, J. T. (1998). Secretion and properties of the large and small lobes of the channel-forming toxin aerolysin. Mol Microbiol 30, 341-352.

Dramsi, S., Biswas, I., Maguin, E., Braun, L., Mastroeni, P. \& Cossart, P. (1995). Entry of Listeria monocytogenes into hepatocytes requires expression of InIB, a surface protein of the internalin multigene family. Mol Microbiol 16, 251-261.

Drevets, D. A., Sawyer, R. T., Potter, T. A. \& Campbell, P. A. (1995). Listeria monocytogenes infects human endothelial cells by two distinct mechanisms. Infect Immun 63, 4268-4276.

Dubail, I., Berche, P., The European Listeria Genome Consortium \& Charbit, A. (2000). Listeriolysin O as a reporter to identify constitutive and in vivo-inducible promoters in the pathogen Listeria monocytogenes. Infect Immun 68, 3242-3250.

Erdenlig, S., Ainsworth, A. J. \& Austin, F. W. (1999). Production of monoclonal antibodies to Listeria monocytogenes and their application to determine the virulence of isolates from channel catfish. Appl Environ Microbiol 65, 2827-2832.

Gaillard, J. L., Berche, P. \& Sansonetti, P. (1986). Transposon mutagenesis as a tool to study the role of hemolysin in the virulence of Listeria monocytogenes. Infect Immun 52, 50-55.

Gaillard, J. L., Berche, P., Mounier, J., Richard, S. \& Sansonetti, P. (1987). In vitro model of penetration and intracellular growth of 
Listeria monocytogenes in the human enterocyte-like cell line Caco-2. Infect Immun 55, 2822-2829.

Gaillard, J. L., Jaubert, F. \& Berche, P. (1996). The inlAB locus mediates the entry of Listeria monocytogenes into hepatocytes in vivo. J Exp Med 183, 359-369.

Geoffroy, C., Gaillard, J. L., Alouf, J. E. \& Berche, P. (1989). Production of thiol-dependent haemolysins by Listeria monocytogenes and related species. J Gen Microbiol 135, 481-487.

Ghani, E., Weis, S., Walev, I., Kehoe, M., Bhakdi, S. \& Palmer, M. (1999). Streptolysin O: inhibition of the conformational change during membrane binding of the monomer prevents oligomerization and pore formation. Biochemistry 38, 15204-15211.

Guex, N., Diemand, A. \& Peitsch, M. C. (1999). Protein modelling for all. Trends Biochem Sci 24, 364-367.

Guzman, C. A., Rohde, M., Chakraborty, T., Domann, E., Hudel, M., Wehland, J. \& Timmis, K. N. (1995). Interaction of Listeria monocytogenes with mouse dendritic cells. Infect Immun 63, 3665-3673.

Heuck, A. P., Hotze, E. M., Tweten, R. K. \& Johnson, A. E. (2000). Mechanism of membrane insertion of a multimeric $\beta$-barrel protein: perfringolysin $\mathrm{O}$ creates a pore using ordered and coupled conformational changes. Mol Cell 6, 1233-1242.

Jacobs, T., Darji, A., Frahm, N., Rohde, M., Wehland, J., Chakraborty, T. \& Weiss, S. (1998). Listeriolysin O: cholesterol inhibits cytolysis but not binding to cellular membranes. Mol Microbiol 28, 1081-1089.

Jacobs, T., Cima-Cabal, M. D., Darji, A. \& 7 other authors (1999). The conserved undecapeptide shared by thiol-activated cytolysins is involved in membrane binding. FEBS Lett 459, 463-466.

Jones, S., Preiter, K. \& Portnoy, D. A. (1996). Conversion of an extracellular cytolysin into a phagosome-specific lysin which supports the growth of an intracellular pathogen. Mol Microbiol 21, 1219-1225.

Kathariou, S., Metz, P., Hof, H. \& Goebel, W. (1987). Tn916induced mutations in the hemolysin determinant affecting virulence of Listeria monocytogenes. J Bacteriol 169, 1291-1297.

Kuhn, M. \& Goebel, W. (1989). Identification of an extracellular protein of Listeria monocytogenes possibly involved in intracellular uptake by mammalian cells. Infect Immun 57, 55-61.

Leimeister-Wachter, M., Haffner, C., Domann, E., Goebel, W. \& Chakraborty, T. (1990). Identification of a gene that positively regulates expression of listeriolysin, the major virulence factor of Listeria monocytogenes. Proc Natl Acad Sci U S A 87, 8336-8340.

Lety, M. A., Frehel, C., Dubail, I., Beretti, J. L., Kayal, S., Berche, P. \& Charbit, A. (2001). Identification of a PEST-like motif in listeriolysin $\mathrm{O}$ required for phagosomal escape and for virulence of Listeria monocytogenes. Mol Microbiol 39, 1124-1140.

Mackaness, G. B. (1962). Cellular resistance to infection. J Exp Med 116, 381-406.

Mengaud, J., Vicente, M. F., Chenevert, J., Pereira, J. M., Geoffroy, C., Gicquel-Sanzey, B., Baquero, F., Perez-Diaz, J. C. \& Cossart, P. (1988). Expression in Escherichia coli and sequence analysis of the listeriolysin $\mathrm{O}$ determinant of Listeria monocytogenes. Infect Immun 56, 766-772.

Michel, E., Reich, K. A., Favier, R., Berche, P. \& Cossart, P. (1990). Attenuated mutants of the intracellular bacterium Listeria monocytogenes obtained by single amino acid substitutions in listeriolysin O. Mol Microbiol 4, 2167-2178.

Nakamura, M., Sekino-Suzuki, N., Mitsui, K. I. \& Ohno-Iwashita, Y. (1998). Contribution of tryptophan residues to the structural changes in perfringolysin $\mathrm{O}$ during interaction with liposomal membranes. J Biochem 123, 1145-1155.

Palmer, M., Harris, R., Freytag, C., Kehoe, M., Tranum-Jensen, J. \& Bhakdi, S. (1998). Assembly mechanism of the oligomeric streptolysin O pore: the early membrane lesion is lined by a free edge of the lipid membrane and is extended gradually during oligomerization. EMBO J 17, 1598-1605.

Park, S. F. \& Stewart, G. S. (1990). High efficiency transformation of Listeria monocytogenes by electroporation of penicillin-treated cells. Gene 94, 129-132.

Portnoy, D. A., Jacks, P. S. \& Hinrichs, D. J. (1988). Role of hemolysin for the intracellular growth of Listeria monocytogenes. J Exp Med 167, 1459-1471.

Renzoni, A., Cossart, P. \& Dramsi, S. (1999). PrfA, the transcriptional activator of virulence genes, is upregulated during interaction of Listeria monocytogenes with mammalian cells and in eukaryotic cell extracts. Mol Microbiol 34, 552-561.

Rossjohn, J., Fell, S. C., McKinstry, W. J., Tweten, R. K. \& Parker, M. W. (1997). Structure of a cholesterol-binding, thiol-activated cytolysin and a model of its membrane form. Cell 89, 685-692.

Shatursky, O., Heuck, A. P., Shepard, L. A., Rossjohn, J., Parker, M. W., Johnson, A. E. \& Tweten, R. K. (1999). The mechanism of membrane insertion for a cholesterol-dependent cytolysin: a novel paradigm for pore-forming toxins. Cell 99, 293-299.

Sheehan, B., Kocks, C., Dramsi, S., Gouin, E., Klarsfeld, A. D., Mengaud, J. \& Cossart, P. (1994). Molecular and genetic determinants of the Listeria monocytogenes infectious process. Curr Top Microbiol Immunol 192, 187-216.

Shepard, L. A., Heuck, A. P., Hamman, B. D., Rossjohn, J., Parker, M. W., Ryan, K. R., Johnson, A. E. \& Tweten, R. K. (1998). Identification of a membrane-spanning domain of the thiolactivated pore-forming toxin Clostridium perfringens perfringolysin $\mathrm{O}$ : an alpha-helical to beta-sheet transition identified by fluorescence spectroscopy. Biochemistry 37, 14563-14574.

Shepard, L. A., Shatursky, O., Johnson, A. E. \& Tweten, R. K. (2000). The mechanism of pore assembly for a cholesteroldependent cytolysin: formation of a large prepore complex precedes the insertion of the transmembrane beta-hairpins. Biochemistry 39, 10284-10293.

Shiba, K. \& Schimmel, P. (1992). Functional assembly of a randomly cleaved protein. Proc Natl Acad Sci USA 89, 1880-1884.

Trieu-Cuot, P., Carlier, C., Poyart-Salmeron, C. \& Courvalin, P. (1990). A pair of mobilizable shuttle vectors conferring resistance to spectinomycin for molecular cloning in Escherichia coli and in Gram-positive bacteria. Nucleic Acids Res 18, 4296.

Trieu-Cuot, P., Carlier, C., Poyart-Salmeron, C. \& Courvalin, P. (1991). An integrative vector exploiting the transposition properties of Tn1545 for insertional mutagenesis and cloning of genes from gram-positive bacteria. Gene 106, 21-27.

Tweten, R. K., Harris, R. W. \& Sims, P. J. (1991). Isolation of a tryptic fragment from Clostridium perfringens theta-toxin that contains sites for membrane binding and self-aggregation. J Biol Chem 266, 12449-12454.

Villanueva, M. S., Sijts, A. J. \& Pamer, E. G. (1995). Listeriolysin is processed efficiently into an MHC class I-associated epitope in Listeria monocytogenes-infected cells. J Immunol 155, 5227-5233.

Received 2 April 2001; revised 4 June 2001; accepted 13 June 2001. 